Three is the Magic Number 1

Group size and therapists' workplace ratings: Three is the magic number

Joshua Patras, Regional Center for Child and Youth Mental Health, UiT The Arctic

University of Norway Trosmø, Norway

Sihu Klest, Regional Center for Child and Youth Mental Health, UiT The Arctic University of Norway, Troms $\emptyset$, Norway 
Summary

The present implementation study compares therapists' workplace ratings of collective efficacy, collaboration, and teamwork in Norwegian child welfare and child psychiatric agencies. Participating therapists were trained in Parent Management Training Oregon model (PMTO) as part of a nationwide implementation of the program. Participating therapists $(\mathrm{N}=$ 83) worked in 67 Norwegian social service agencies. Respondents were part of three conditions: (1) PMTO therapists working in agencies with no other PMTO therapists $(\mathrm{N}=$ 45), (2) two PMTO therapists working together in the same agency $(\mathrm{N}=23)$, and (3) three therapists working together in the same agency $(\mathrm{N}=15)$.

Findings

Therapists working in clusters of 3 rated their workplaces more positively than therapists who worked in agencies with 1 or 2 PMTO interventionists, $\beta=.29, p=.03$; Cohen's $d=.80$. The result was independent of agency size, leadership quality, service population, and therapist education. There was no difference in workplace ratings between PMTO therapists working in agencies in pairs (2 PMTO therapists) and those working in agencies alone (1 PMTO therapist), $p=.45$.

Applications

PMTO therapists working in clusters of three appeared to have better experiences with their workplace than therapists with no other PMTO colleagues, and those with only one other PMTO colleague. This result suggests that clustering therapists together in groups of at least three may create a better work environment for therapists using newly-implemented interventions.

Keywords: Implementation effectiveness, Mental health organizations, Evidence-based practice 
Group Size and Therapists' Workplace Ratings: Three is the Magic Number

As evidence-based programs (EBPs) move from controlled settings into the complex, uncontrolled environments of service organizations, it is important to identify effective methods for program implementation in order to aid intervention success. The present study examines the advantage of clustering two or three EBP therapists in the same social services agency during a nationwide implementation of an intervention. We discuss the possibility that clustering therapists at the same agency may be a straightforward and effective method to facilitate successful program implementation.

In recent years, implementation researchers have identified key areas that require further study to better understand the processes that facilitate or impede implementation of evidence-based practices (Fixsen, Blase, Naoom, \& Wallace, 2009; Fixsen, Naoom, Blase, Freidman, \& Wallace, 2005; Greenhalgh, Robert, Macfarlane, Bate, \& Kyriakidou, 2004; Proctor et al., 2009). Programs shown to be effective in laboratory settings often lose effectiveness when moving from academic settings into real world practice. Because these drops in effectiveness co-occur with moving from the laboratory into the community, researchers have focused on characteristics of the implementing organizations as a way to explain these differential results (Burns, Hoagwood, \& Mrazek, 1999; Rosenheck, 2001; Rye \& Kimberly, 2007). Some evidence exists that suggests positive workplace culture and climate in social service agencies can influence the treatment effectiveness of evidence-based practices by cultivating an implementation environment that is more open and receptive to the intervention (Glisson, 2002, 2007). For example, Glisson and Hemmelgarn (1998) found that social service organizations with more positive climates provided better service quality and showed better outcomes for clients. Other studies have shown that social support in the workplace can be important to employee job satisfaction and retention rates. Corrigan, 
Holmes, and Luchins (1995) found that employees working in psychiatric hospitals who rated collegial support higher were less likely to report symptoms of burnout than employees who reported that they experienced less collegial support. The aim of this study is to identify a relatively simple implementation method that may impact therapist perceptions of the existing organizational context of their agencies.

\section{Project Background}

In 1998, the Norwegian government began a nationwide implementation of Parent Management Training Oregon (PMTO) model, an evidence-based intervention aimed at reducing behavior problems in children and youth, within Norwegian child welfare and child psychiatric agencies. Families with a child between the ages of four and twelve who contacted a social services agency for help with their child's conduct disorder were eligible to receive PMTO treatment. The current study is part of a larger project following the Norwegian implementation of PMTO with a focus on identifying organizational characteristics of social service agencies that influence program effectiveness. Detailed information about bringing PMTO to Norway and the implementation process can be found in Ogden, Forgatch, Askeland, Patterson, \& Bullock (2005). Results of a randomizedcontrolled effectiveness trial of PMTO in Norway can be found in Ogden and Amlund-Hagen (2008).

Norwegian therapists trained in the PMTO method worked with PMTO mentors outside of their agencies for at least one year and were among the first evidence-based intervention practitioners to work in Norwegian social service agencies (Ogden et al., 2005). Once trained, PMTO therapists continued to work as part of a group of general therapists at social service agencies, most of whom continued practicing treatment as usual. Practicing PMTO therapists received small group booster-trainings outside of their agencies 
approximately two times per year and were able to contact PMTO experts for support at any time.

PMTO was somewhat unique among treatment programs in use in Norway, in that most other treatments in the social service agencies were not evidence-based. This presented implementation teams, as well as implementing therapists, with the challenge of acceptance of PMTO in the social services community in Norway. Many PMTO therapists met with resistance from their coworkers for using a method that was thought to be too "manualbased", and as a result, there was concern that the implementation therapists may be less satisfied in their jobs (which could be a threat to program sustainability). The PMTO implementation team in Norway believed that it may be an important implementation strategy to cluster therapists in groups at each implementation site in order to produce better treatment results and lower therapist burnout (T. Christiansen, personal communication, April, 2008).

There is evidence in the behavioral literature that individuals have a need to interact with others who share similar experiences and values, and to whom they can relate and form interpersonal attachments (Baumeister \& Leary, 1995). In the organizational literature, this is referred to as collective identity; it is the idea that individuals can relate to and value the group of which they are a member, even if interpersonally there is no strong attraction between group members (Brewer \& Gardner, 1996). Molm, Collett, and Schaeffer (2006) report that the more actors you have working in an informal system of exchange, the greater their feelings of solidarity. In order for perceptions of trust and greater social unity to exist in relation to the execution of generalized reciprocity in an informal system, it is necessary to have at least three actors (Molm et al., 2006). Because the aims of the PMTO program are specified and likely unique within service organizations at the time, PMTO therapists can be viewed as a sub-group within a larger organization. Furthermore, PMTO therapists who work together report that they are able to assist one another with difficult cases, discuss effective 
approaches to therapy, share work tasks when needed, and offer other forms of moral and professional support in an informal way (Patras \& Klest, 2009). Klest (2014) found that PMTO therapists who worked with other PMTO colleagues were more likely to continue working with PMTO, treated more clients, and were less likely to quit using PMTO than other PMTO therapists.

Based on the experiences of the PMTO implementation team, as well as work-group theory, we hypothesized that PMTO therapists who work at agencies with progressively more PMTO colleagues would rate their workplaces (constructed from collective efficacy, collaboration, and quality of teamwork) progressively higher. The maximum number of PMTO therapists working in any agency was three. Because group theory suggests that three is a critical number, especially in relation to reciprocity, and due to limited sample size, we specified two contrasts to simultaneously test two hypotheses using one model (Molm et al., 2006).

Hypotheses: 1) PMTO therapists who work in agencies with two PMTO therapists will rate their workplaces higher than those in agencies with one PMTO therapist. 2) PMTO therapists who work in groups of three will rate their workplaces more positively than PMTO therapists who work in agencies with one or two PMTO therapists. The model includes four control variables: 1) total number of employees at the organization, 2) size of the service population, 3) therapist education level, and 4) therapist ratings of agency leadership.

\section{Methods}

\section{Participants}

Every PMTO therapist in Norway, at the time of the present study, 138 PMTO therapists were invited to participate, 83 agreed. A workplace survey was sent to the therapists who agreed to participate $(N=83)$. We were not able to identify data that would allow us to compare the characteristics of the therapists who participated in the study and 
those who did not. Of the participating therapists, 45 worked in agencies with one PMTO therapist, 23 worked in agencies with two PMTO therapists, and 15 worked in agencies with three PMTO therapists; no agencies in this study had more than three PMTO therapists at the time of the study. Surveys were returned from $100 \%$ of therapists who agreed to participate. Of the responding PMTO therapists, $80 \%$ were female and $20 \%$ were male; $51 \%$ had a bachelor or professional degree, $41 \%$ had a master's degree, civil servant degree, or some graduate school, $1 \%$ had a $\mathrm{PhD}$, and $7 \%$ did not complete the questions detailing their education level. Protocols from human subjects' review boards in Norway and the United States were followed during the study.

\section{Measures}

The study survey included questions about the workplace environments in therapists' organizations, the therapists' backgrounds, and information about the area that they served. Three scales of agency environment, 1) collective efficacy, 2) collaboration, and 3) teamwork, were constructed based on results from exploratory and confirmatory factor analyses.

The collective efficacy measure focused on the therapists' perceptions of the quality and effectiveness of the work performed by themselves and their colleagues (Patras \& Klest, 2013). The scale is comprised of 6-items $(\alpha=.78)$ and was rated on a 5-point scale from 1 "Completely disagree" to 5 "Completely agree." . Example questions include: "Employees in this organization are able to reach difficult clients," and "Employees here can help change parent-child interaction."

The collaboration scale, taken from the Supervisor Support questionnaire (Bullock, 2003), aims to measure collaborative interaction between employees within the organization. The 4-items $(\alpha=.68)$ were rated on a 5-point scale from 1 "Strongly disagree" to 5 "Strongly agree." Example questions include: "We have regular team meetings where we discuss our work with children and families" and "Employees collaborate to find effective strategies in 
their work with clients." These items were intended to target work conducted within the agency rather than in outside trainings; PMTO therapists work within the context of their agencies rather than in separate units and they participate in the normal activities of their agencies.

Therapist ratings of Teamwork in the workplace, an 8-item scale $(\alpha=.86)$, was taken from the Readiness for Organizational Learning and Evaluation (ROLE) instrument (Preskill $\&$ Torres, 2000). Questions were answered on a 5-point scale from 1 "Strongly disagree" to 5 "Strongly agree." Example questions from the Teamwork scale: "When conflict arises among the team members, it is resolved effectively" and "Team meetings are well facilitated."

The exogenous predictor variables in the model include two Helmert contrasts specifying the number of PMTO therapists working at the agency, as well as four control variables: 1) total number of employees at the organization, 2) size of the agency's service populations 3) therapist education level, and 4) therapist ratings of agency leadership. Number of employees at the agency was taken from the workplace questionnaire and was coded on an ordinal scale consisting of eight unequal bin sizes ( 1 - "1 to 5 employees," 2 "6 to 10 employees," 3 "11 to 20 employees," 4 "21 to 30 employees," 5 “31 to 40 employees," 6 "41 to 50 employees," 7 "51 to 70 employees," and 8 "More than 70 employees"). Service population was taken from the workplace questionnaire and was coded on an ordinal scale consisting of six unequal bin sizes (1 "Less than 1000," 2 "1000 to 5000," 3 "5000 to 10000 ," 4 “10000 to 50000," 5 "50000 to 100000," and 6 "More than 100000"). Therapist self-report of education level was coded to represent two levels of education (1 "Bachelors or other professional degree," 2 “Masters degree or equivalent, some graduate school, or $\mathrm{PhD}$ ”).

\section{Analysis}

We fit a factor structural equation model (SEM) in Amos version 16.0 (Arbuckle, 2007) in order to assess therapists' ratings of workplace based on the number of therapists at 
the agency, while controlling for the hypothesized covariates. Conducting the analysis using SEM rather than analysis of variance allowed us to control for size of organization, service population, therapist education, and leadership quality as well as use a latent construct of workplace as the outcome variable. Full information maximum likelihood (FIML) estimation of missing data was employed. Using FIML provides for more robust model estimates than traditional methods of missing data imputation such as mean, regression, or imputation using EM algorithm (Graham, 2009). A missing-values analysis provided support that all of the data was missing completely at random, Little's MCAR test: $\chi^{2}(8)=4.46, p=.81$.

The model included two Helmert contrast independent variables: 1) comparing ratings from PMTO therapists working individually with ratings from PMTO therapists working with one other PMTO therapist, and 2) comparing the ratings of PMTO therapists working in clusters of three with the combined ratings of PMTO therapists working individually and in pairs. There were no agencies in the study with more than three PMTO therapists. The two contrast variables, ‘1 vs. 2 PMTO’ and '1 \& 2 vs. 3 PMTO’ represented orthogonal dummy coded variables (see Table 1). Using this coding method, the two orthogonal contrasts allowed for simultaneous testing of both hypotheses. A positive and statistically significant direct effect for the ' 1 vs. 2 PMTO' variable would indicate that therapists working at agencies with two PMTO therapists rate their workplace more positively than those working alone, while a positive and significant direct effect for the ' $1 \& 2$ vs. 3 PMTO' variable would indicate that therapists who work with three PMTO therapists rate their workplace more positively than the combined ratings of one and two PMTO therapists. The dependent variable was a latent factor of three workplace scales: collective efficacy, collaboration, and teamwork. 
We controlled for: 1) total number of employees at the organization, 2) size of the service population, 3) therapist education level, and 4) therapist ratings of agency leadership. Leadership was chosen as a covariate because prior research has shown leadership to be correlated with organizational variables such as collective efficacy. For example, Chen and Bliese (2002) found that leadership climate in military organizations was a predictor of organizational level collective efficacy.

A single-level analytic approach was used to analyze this data rather than a multilevel approach. Although therapists were nested within agencies, recommendations for the number of cases within each group (e.g., agencies) necessary to attain valid and reliable group-level estimates are, at minimum, two observations per group on average (Clarke, 2008). The average number of therapists within each agency in our sample was 1.24. To employ a multilevel approach with such small group membership could lead to an over estimate of group-level random effects (Clarke, 2008). Therefore, to avoid overestimation of the random effects at level 2, a single-level approach was utilized.

\section{Results}

The model (see Figure 1), including two predictors, four control variables, and a latent workplace outcome variable, obtained excellent fit to the data in $\chi^{2}(13)=6.37, p=.93$, comparative fit index $[\mathrm{CFI}]=1.00$, root mean square error of approximation $[\mathrm{RMSEA}]=.00$.

\section{Insert Figure 1 about here}

There was a statistically significant direct effect for the contrast variable ' $1 \& 2$ vs. 3 PMTO'; PMTO therapists working in clusters of three in the same agencies rated their workplaces significantly higher than the aggregate ratings of those in agencies with 1 or 2 PMTO 
therapists, $\beta=.29, p=.03$. There was no statistically significant direct effect for the workplace ratings of PMTO therapists who worked in agencies with one therapist compared to those in agencies with two therapists, $\beta=.10, p=.45$. Descriptive statistics for the observed and factor means were computed for each of the three participant groups (see Table 2).

Effect sizes for the statistically significant effect of three PMTO therapists working in the same agency compared to the aggregate scores of one and two PMTO therapists were calculated for the three observed scales of the workplace factor: collective efficacy $d=.75$, collaboration $d=.79$, and teamwork $d=.54$. These effect sizes are medium to large. An effect size for the latent factor was also computed based on the contrast between three PMTO therapists and the aggregate scores of one and two PMTO therapists, Cohen's $d=.80$ (see Table 3); this is a large effect size.

\section{Discussion}

Our first hypothesis was not supported; there were no significant differences in ratings between PMTO therapists who do not work with other PMTO interventionists and those who work with one other PMTO colleague. Our second hypothesis was supported; PMTO therapists who worked in agencies with three trained practitioners rated their workplaces more positively than PMTO therapists who worked in agencies with one or two PMTO therapists. The results from the first hypothesis did not support the second hypothesis. However, taken together, the results are notable because they suggest that the mechanism for the findings is not simply one of loneliness versus togetherness, but rather that there is a social process that may only occur when there are a critical number of individuals in the group. Molm, Collett, and Schaeffer's (2006) work describing the requirement of forming a group comprised of at least three individuals for generalized reciprocity to exist, supports this supposition. The result is particularly interesting because all PMTO therapists have program support available 
to them at any time from PMTO trainers outside of their agencies and all practitioners meet with trainers in groups with about eight other practitioners to discuss cases and receive guidance approximately six times per year (PMTO therapists do not receive supervision or support within their agencies that is specific to PMTO). Those practitioners who work together in the same agencies report assisting one another informally with difficult cases, sharing work tasks, and approaching management to negotiate problems they face in working with the program within the existing structure of the agency (e.g., therapists may not be assigned appropriate families for PMTO treatment, Patras \& Klest, 2009). If there is more than one colleague available to therapists, the support they receive may have more breadth and be more readily available (e.g., if one colleague is absent, the other may be available). In addition, a group of three practitioners may gain more acceptance from colleagues not associated with their program as well as receive more support from their agencies than two practitioners.

\section{Limitations}

One limitation of the present study is that we were not able to test organizations with more than three PMTO therapists; therefore, it is not known whether or not adding more practitioners working with the same program would produce a linear increase in workplace ratings. We suspect that the dramatic increase in ratings that was observed between two practitioners in the organization and three practitioners would not be found between three practitioners and four or more practitioners, but would begin to level off at three with more modest increases in ratings as more practitioners were introduced. This would likely depend on other contextual factors of the organization, such as agency size.

\section{Conclusions}

In future research we plan to test the effects of increasing the number of practitioners in an organization working with the same program in an effort to identify the optimal number 
of EBP therapists needed for positive implementation outcomes. It is also important to directly test program outcomes in future research to determine whether clustering at least three practitioners trained in the same method improves retention rates (both practitioner and client), treatment fidelity, treatment efficacy, and program sustainability. We are currently working on a follow-up study to examine the relationship between some outcome variables of PMTO implementation (e.g., number of cases treated in the past six months) and the number of therapists in the agency.

In addition, future research should address limitations of the present study. The group sizes for the different clusters of therapists were unequal; there were only 15 practitioners from 5 agencies who worked in organizations with three PMTO therapists while there were 45 who worked alone. Another limitation of the present study is that it was not a randomizedcontrolled trial, therefore it is not possible to identify a causal relationship between the factors; randomly assigning specific numbers of practitioners to agencies at the beginning of an intervention is the ideal method for follow-up research to address this issue. If conducting an RTC is not feasible, other study design methods could help to address this issue.

Collecting data from agencies and practitioners both before and after program training would allow researchers to control for baseline differences in agency ratings, and collecting data from a larger sample could allow for testing mediation and moderation effects.

Our model controlled for four potentially meaningful relationships which might have served as an explanation for the outcome. Although the number of employees and the size of the service population were significantly correlated with the number of PMTO therapists at the agency, as one might expect, neither condition explained the relationship between the number of therapists and their workplace ratings. Leadership was highly predictive of workplace ratings, but did not serve to explain the observed relationship between differing numbers of PMTO therapists and their ratings of the workplace. Finally, therapist education 
was neither highly correlated with the number of PMTO therapists at the agency, nor statistically significantly predicted differential ratings of the workplace. In sum, the inclusion of the four control variables did little to explain the higher ratings of therapists who work in clusters of three. It is possible, however, that systematic differences exist in organizations other than those that were measured and controlled for. Future research should examine whether other organizational and contextual variables could help to explain this result.

Although previous literature has suggested that group membership is important to feelings of solidarity, to the best of our knowledge, no study has specifically examined the effect of working in a group with colleagues trained in the same EBP on ratings of workplace or on performance outcomes. We believe this to be an interesting finding that has the potential to be beneficial to program implementation outcomes, particularly given the large effect size of the result. We would like to encourage further inquiry and development of this topic from researchers and practitioners. Evidence-based program implementation efforts are time consuming and costly. It is challenging to sustain programs and maintain the effectiveness of interventions in real-world environments. Therefore, identifying relatively simple procedures that may aid implementation efforts could be of great value.

\section{Ethics}

Human subjects' protocols from The Norwegian National Committee for Research Ethics and the Norwegian Social Science Data Registry were followed. In addition, the Internal Review Board for research with human subjects at Oregon Social Learning Center in the United States approved the study prior to data collection.

\section{Acknowledgements}

The authors would like to acknowledge Marion Forgatch and Terje Ogden as principal investigators of the PMTO program in Norway. 
Three is the Magic Number 15

\section{Funding}

This work was supported by the Prevention Research Branch, NIDA, US PHS (grant no.

RO1 DA 16097). Data used in this study are available from the authors. 


\section{References}

Arbuckle, J. L. (2007). Amos (Version 16.0) [Computer Program]. Chicago: SPSS.

Baumeister, R.F., \& Leary, M.R. (1995). The need to belong: Desire for interpersonal attachments as a fundamental human motivation. Psychological Bulletin, 117(3), 497529. doi: 10.1037/0033-2909.117.3.497

Brewer, M.B., \& Gardner, W. (1996). Who is this "we"? Levels of collective identity and self representations. Journal of Personality and Social Psychology, 71(1), 83-93. doi: $10.1037 / 0022-3514.71 .1 .83$

Bullock, B. (2003). Supervisor Support. Eugene, OR: Oregon Social Learning Center.

Burns, B.J., Hoagwood, K., \& Mrazek, P.J. (1999). Effective treatment for mental disorders in children and adolescents. Clinical Child and Family Psychology Review, 2(4), 199254. doi: 10.1023/a:1021826216025

Chen, G., \& Bliese, P.D. (2002). The role of different levels of leadership in predicting selfand collective efficacy: Evidence for discontinuity. Journal of Applied Psychology, 87(3), 549-556. doi: 10.1037/0021-9010.87.3.549

Clarke, P. (2008). When can group level clustering be ignored? Multilevel models versus single-level models with sparse data. Journal of Epidemiology and Community Health, 62(8), 752-758. doi: 10.1136/jech.2007.060798

Corrigan, P.W., Holmes, E.P., \& Luchins, D. (1995). Burnout and collegial support in state psychiatric hospital staff. Journal of Clinical Psychology, 51(5), 703-710.

Fixsen, D.L., Blase, K.A., Naoom, S.F., \& Wallace, F. (2009). Core implementation components. Research on Social Work Practice, 19(5), 531-540. doi: $10.1177 / 1049731509335549$

Fixsen, D.L., Naoom, S.F., Blase, K.A., Freidman, R.M., \& Wallace, F. (2005). Implementation research: A synthesis of the literature. (FMHI Publication \#231). 
University of South Florida, Louis de la Parte Florida Mental Health Institute, National Implementation Research Network.

Glisson, C. (2002). The organizational context of children's mental health services. Clinical Child and Family Psychology Review, 5(4), 233-253. doi: 10.1023/a:1020972906177

Glisson, C. (2007). Assessing and changing organizational culture and climate for effective services. Research on Social Work Practice, 17(6), 736-747. doi:

\section{$10.1177 / 1049731507301659$}

Glisson, C., \& Hemmelgarn, A. (1998). The effects of organizational climate and interorganizational coordination on the quality and outcomes of children's service systems. Child Abuse \&amp; Neglect, 22(5), 401-421. doi: 10.1016/s01452134(98)00005-2

Graham, J.W. (2009). Missing data analysis: Making it work in the real world. Annual Review of Psychology, 60, 549-576. doi: 10.1146/annurev.psych.58.110405.085530

Greenhalgh, T., Robert, G., Macfarlane, F., Bate, P., \& Kyriakidou, O. (2004). Diffusion of innovations in service organizations: Systematic review and recommendations. Milbank Quarterly, 82(4), 581-629. doi: 10.1111/j.0887-378X.2004.00325.x

Klest, S. K. (2014). Clustering practitioners within service organizations may improve implementation outcomes for evidence-based programs. Zeitschrift für Psychologie, 222(1), 30-36. doi: 10.1027/2151-2604/a000163

Molm, L.D., Collett, J.L., \& Schaefer, D.R. (2006). Conflict and fairness in social exchange. Social Forces, 84(4), 2331-2352. doi: 10.1353/sof.2006.0100

Ogden, T., Forgatch, M.S., Askeland, E., Patterson, G.R., \& Bullock, B.M. (2005). Implementation of parent management training at the national level: The case of norway. Journal of Social Work Practice, 19(3), 317-329. doi: $1080 / 02650530500291518$ 
Ogden, T., \& Hagen, K.A. (2008). Treatment effectiveness of parent management training in norway: A randomized controlled trial of children with conduct problems. Journal of Consulting and Clinical Psychology, 76(4), 607-621. doi: 10.1037/0022006X.76.4.607

Patras, J. \& Klest, S. (2009). Program implementation: The benefits of working in groups. Paper presented at the $11^{\text {th }}$ Annual European Congress of Psychology, Oslo, Norway.

Patras, J., \& Klest, S.K. (2013). Development of a collective efficacy measure for use in social service organizations. Journal of Social Work, 13(1), 96-106. doi: $10.1177 / 1468017311412034$

Preskill, H. \& Torres, R. (2000). The Readiness for Organizational Learning and Evaluation Instrument (ROLE) Version 4. University of New Mexico, Albuquerque. http://www.fsg.org/Portals/0/Uploads/Documents/ImpactAreas/ROLE_Survey.pdf

Proctor, E.K., Landsverk, J., Aarons, G., Chambers, D., Glisson, C., \& Mittman, B. (2009). Implementation research in mental health services: An emerging science with conceptual, methodological, and training challenges. Administration and Policy in Mental Health and Mental Health Services Research, 36(1), 24-34. doi: $10.1007 / \mathrm{s} 10488-008-0197-4$

Rosenheck, R.A. (2001). Organizational process: A missing link between research and practice. Psychiatric services, 52(12), 1607-1612. doi: 10.1176/appi.ps.52.12.1607

Rye, C.B., \& Kimberly, J.R. (2007). The adoption of innovations by provider organizations in health care. Medical Care Research and Review, 64(3), 235-278. doi:

$10.1177 / 1077558707299865$ 
Table 1

Coding of Contrast Variables

\begin{tabular}{lcc}
\hline $\begin{array}{l}\text { Number of } \\
\text { PMTO Therapists }\end{array}$ & $\begin{array}{c}\text { Helmert } \\
\text { 1 vs. } 2\end{array}$ & $\begin{array}{c}\text { Helmert } \\
1 \& 2 \text { vs. 3 }\end{array}$ \\
\hline 1 & -1 & -.5 \\
2 & 1 & -.5 \\
3 & 0 & 1 \\
\hline
\end{tabular}


Table 2

Descriptive Statistics of Scale Scores and Factor Means by Response Group

\begin{tabular}{|c|c|c|c|c|c|c|}
\hline & \multicolumn{2}{|c|}{1 PMTO } & \multicolumn{2}{|c|}{2 PMTO } & \multicolumn{2}{|c|}{3 PMTO } \\
\hline & Mean & $\begin{array}{c}\text { Std. } \\
\text { Deviation }\end{array}$ & Mean & $\begin{array}{c}\text { Std. } \\
\text { Deviation }\end{array}$ & Mean & $\begin{array}{c}\text { Std. } \\
\text { Deviation }\end{array}$ \\
\hline $\begin{array}{l}\text { Collective } \\
\text { Efficacy }\end{array}$ & 3.84 & .58 & 3.87 & .57 & 4.24 & .47 \\
\hline Collaboration & 3.67 & .78 & 3.66 & .53 & 4.17 & .57 \\
\hline Teamwork & 3.69 & 66 & 3.85 & .45 & 4.08 & .65 \\
\hline $\begin{array}{l}\text { Workplace } \\
\text { (Factor mean) }\end{array}$ & -.22 & 1.01 & -.04 & .68 & .63 & 1.08 \\
\hline
\end{tabular}


Table 3

Estimated Effect Sizes for 3 PMTO Therapists Versus the Combined Mean of 1 and 2 PMTO Therapists

\begin{tabular}{|c|c|c|c|c|c|}
\hline & $\begin{array}{l}\text { Contrast } \\
\text { Groups }\end{array}$ & Mean & $\begin{array}{l}\text { Std. } \\
\text { Deviation }\end{array}$ & $\begin{array}{c}\text { Cohen's } \\
d\end{array}$ & $\begin{array}{l}\text { Effect } \\
\text { size } r\end{array}$ \\
\hline \multirow{2}{*}{$\begin{array}{l}\text { Collective } \\
\text { Efficacy }\end{array}$} & $1 \& 2$ PMTO & 3.85 & .57 & \multirow[t]{2}{*}{.75} & \multirow[t]{2}{*}{.35} \\
\hline & 3 PMTO & 4.24 & .47 & & \\
\hline \multirow[t]{2}{*}{ Collaboration } & $1 \& 2$ PMTO & 3.67 & .70 & \multirow[t]{2}{*}{.78} & \multirow[t]{2}{*}{.36} \\
\hline & 3 PMTO & 4.17 & .57 & & \\
\hline \multirow[t]{2}{*}{ Teamwork } & $1 \& 2$ PMTO & 3.74 & .61 & \multirow[t]{2}{*}{.53} & \multirow[t]{2}{*}{.26} \\
\hline & 3 PMTO & 4.08 & .65 & & \\
\hline \multirow{2}{*}{$\begin{array}{l}\text { Workplace } \\
\text { (Factor mean) }\end{array}$} & $1 \& 2$ PMTO & -.17 & .92 & \multirow[t]{2}{*}{.80} & \multirow[t]{2}{*}{.37} \\
\hline & 3 PMTO & .63 & 1.08 & & \\
\hline
\end{tabular}


Three is the Magic Number 22

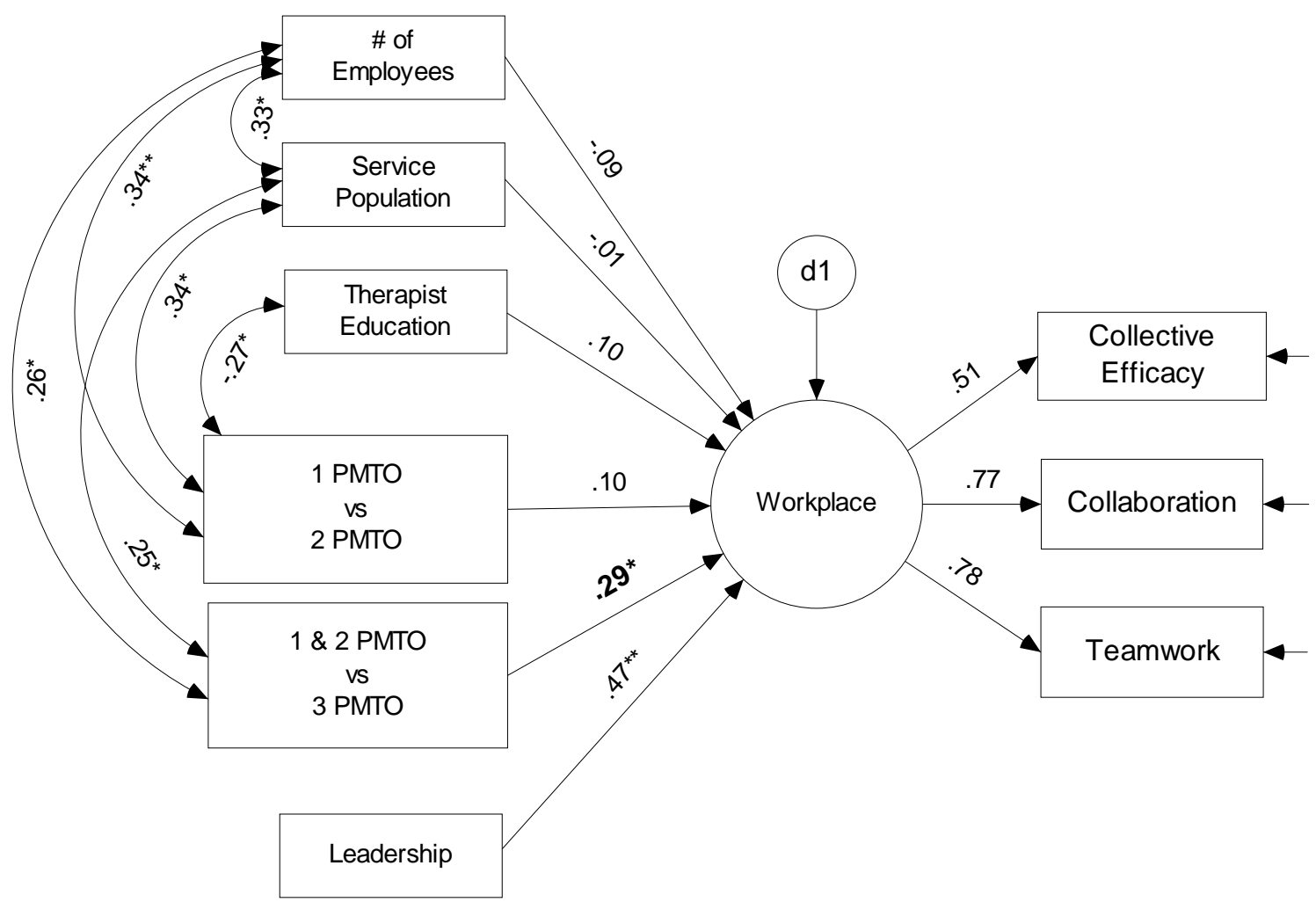


Three is the Magic Number 23

\section{Captions:}

Figure 1

Number of PMTO Therapists Predicting Ratings of Workplace Controlling for Leadership, Number of Employees, Service Population Size, and Therapist Education

$\chi^{2}(13)=6.37, p=.93, \mathrm{CFI}=1.00, \mathrm{RMSEA}=.00 . * p<.05, * * p<.01$

Note: All exogenous variables except the two contrast codes were allowed to covary; nonsignificant covariances omitted from the model to improve readability 